\title{
EDUCACIÓN PARA EL NUEVO SIGLO
}

\section{Education for the new century}

Los rápidos cambios en el mundo digital exigen una nueva mirada a la educación en general, y en particular hacia la educación en salud. Las competencias que requerimos formar desde la educación pre-básica hasta la educación secundaria y luego las requeridas para la educación superior, deben encontrar un eco en las nuevas tendencias y hacerse partícipe de la evoluciónn tecnológica que hoy es parte integral de todos los jóvenes desde sus primeros años de vida. Aprovechar o adaptarse a la forma de comunicación que para ellos es y será cada vez mayor, constituye un poderoso denominador en la ecuación.

Hemos comentado y puesto énfasis en innumerables oportunidades en la enseñanza centrada en el alumno, pero esto muchas veces pasa de ser un enunciado deseable sin que se lleve a la práctica. Esto obedece a que se requiere un cambio paradigmático, que exige un esfuerzo formativo por parte de los docentes y que como todo cambio, provoca resistencia para adoptar nuevas actitudes. Sólo quisiera referirme a algunos aspectos puntuales que me parecen esenciales para entender estos cambios. En primer lugar una interesante experiencia que ha presentado Eric Mazur de Harvard en varias conferencias y que se refiere a la medición de la actividad cerebral mediante un dispositivo portátil, que los estudiantes universitarios llevan durante algunas semanas registrando lo que ocurre durante el sueño, durante horas de estudio, asistencia a laboratorios, viendo TV, asistiendo a clases, horas de relajo, estudiando y dando pruebas, entre otras. Se aprecia que la actividad cerebral es más plana durante las clases y sólo comparable a cuando miran televisión. Aquí está la raíz del cambio que significa migrar de una clase, que es sólo la simple transferencia de contenidos desde la presentación del profesor a los cuadernos del alumno sin pasar por el cerebro para su procesamiento. Esto es lo que ha llevado a muchos a acuñar el concepto de flip the class room (invierte la sala de clases). Esto implica usar todos los medios tecnológicos posibles en la actualidad (acceso electrónico a bibliotecas, videos de clases, contenidos en You Tube, etc.) para que los alumnos adquieran el conocimiento en las horas, ritmo y ubicación que más les acomoden y vengan a la sala de clases para discutir sus hallazgos, especular respecto a las aplicaciones y muy importante discutir en grupos respecto al problema. El profesor por lo tanto pasa a tener un rol muy diferente y que es el de moderar, aclarar, guiar, etc. Esto nos lleva a otro concepto que es de instrucción por pares o peer instruction, enseñanza por pares, que permite a estudiantes más aventajados o que han podido captar mejor los contenidos adquiridos a través de su trabajo personal, ayudar a quienes tuvieron más dificultad en comprenderlos. Esto no solo implica un cambio de actitudes en al alumno y profesor, requiere además un cambio no menor de infraestructura para la educación. Los grandes auditorios, o salas de clases con sillas en hileras, deberán dar paso a espacios en donde se puedan organizar mesas de trabajo grupal adaptables para 4, 6, 8 ó 10 alumnos, rodeados por pantallas multimedia y utilizando notebooks, netbooks, tablets, teléfonos inteligentes, etc. Es un mundo fascinante que se nos abre por delante donde el principal escollo será sin lugar a dudas la resistencia al cambio tanto de los profesores y no nos equivoquemos, también de los estudiantes. Estimulo a quienes están interesados en estos tópicos que revisen el tema revisando a autores como Eric Manzur, Salman Khan y Sugata Mitra entre otros por You Tube o conferencias TED, para hacer el honor al uso de las nuevas tecnologías y medios de comunicación.

El segundo aspecto que me gustaría abordar es una reflexión acerca de cómo aproximarse con una nueva mirada a la enseñanza de las ciencias o profesiones de la salud en este siglo por el cual hemos comenzado a transitar. Es común citar el informe Flexner que cumplió ya más de cien años y es probable que la estructura curricular rígida, sin integración, siga siendo aplicada por muchas escuelas de Medicina, con agregados tales como Aprendizajes Basados en Problemas, Simulación, etc. No cabe duda que estos dos últimos aspectos constituyen cambios significativos, desde la segunda mitad del siglo pasado, permitiendo importantes labores de integración y aplicación de conocimientos, habilidades y actitudes. La seguridad, privacidad y derechos de los pacientes comenzaron a ser más valoradas y protegidas, mediante la adquisición de habilidades en centros o ambientes de entrenamiento que permiten la repetición de destrezas tanto técnicas como sociales, hasta poder comprobar su correcta ejecución y aplicación. Todo esto es coherente con el avance metodológico y tecnológico en la enseñanza. 
Sin embargo, hay elementos que debemos tomar en cuenta al repensar la educación médica y de ciencias de la salud en el presente siglo. Hoy vivimos en una sociedad cuyos actores tienen una expectativa de vida que es el doble de lo que se tenía en la mitad del siglo pasado. Así mismo sabemos que los ciudadanos están más informados gracias al acceso universal a los medios digitales. Esto los transforma en pacientes más empoderados. Hay que recalcar que hay una mayor conciencia de la significación de un estilo de vida saludable, con conocimiento de principios de nutrición sana y la valoración de la actividad fisica. Paralelamente se producen cambios epidemiológicos importantes con la irrupción de nuevos agentes patógenos como el HIV y otros y una tendencia a la sobre especialización, que puede tener su raiz en el rápido recambio del conocimiento que deja al médico general, aquel que se ocupaba de la medicina familiar a un paso de su extinción o en la imposibilidad de abarcar en profundidad muchas áreas.

Dos cambios importantes que deben marcar la tendencia en educación en salud, serían el desplazamiento de los centros de práctica desde los hospitales a la atención primaria y al concepto de red. Hoy día los hospitales han evolucionado de modo que una importante proporción de sus camas se dedican a enfermos críticos, incluso con alta especialización tal como, intensivo general, unidades coronarias y de cuidados intermedios, unidades neuro-críticas, etc. Este ambiente ya no es el ideal para la formación de pregrado para nuestros médicos y equipos de salud, ya que aparte de representar el fracaso de la medicina preventiva, por su alta complejidad deben estar reservadas para la formación de especialistas en las áreas pertinentes. Uno de los postulados es la concentración de nuestros estudiantes en los sitios de atención primaria y que puedan ver la integración del sistema, desde el trabajo en terreno con las familias y comunidades, hasta la necesidad de acompañar al individuo hasta el centro crítico. En parte hemos vivido un modesto desplazamiento de los sitios de práctica en hospitales a los centros de atención primaria, pero esto debe intensificarse y concordar con las políticas de salud, para hacer coherente esta iniciativa. Ello requiere una planificación conjunta con los actores claves, gobierno, universidades, sociedades cientificas, asociaciones gremiales y la comunidad para elaborar una estrategia común e incluso ordenar la asignación de recursos para asegurar que el objetivo de las políticas en educación y las sanitarias coincida con el objetivo final, que es acceso igualitario y universal a la salud.

Un segundo aspecto importante es la formación interprofesional, para adquirir las competencias de trabajo en equipo desde los inicios de su formación. Hoy cada profesión desarrolla su curriculum en una forma independiente y aislada con barreras importantes para la integración entre sí. Sin embargo una de las características fundamentales del desarrollo y desempeño profesional una vez recibidos, será el trabajo en equipo y para ello no los preparamos. Es necesario que coincidiendo en algunas actividades que concuerden en la expresión de competencias comunes, puedan comprender sus roles como agentes de cambio, opciones de liderazgo del más apto y por sobre todo un marco ético y de transparencia que son elementos que impregnan este nuevo siglo.

Tomando en cuenta estos dos enunciados, coincido con las propuestas elaboradas por el de Julio Frenk, Decano de la Harvard School of Public Health y un amplio número de pensadores y publicado en Lancet el año 2010. Podemos esquematizar las etapas de este complejo proceso en tres niveles:

1. Aprendizaje Informativo: entendiendo como tal el proceso de adquirir conocimientos y habilidades centrando la metodología a usar en los aspectos ya señalados con anterioridad. Dentro de estos es esencial el trabajo activo e interactivo para el desarrollo de las capacidades de razonamiento y análisis crítico, que permitan aplicar conceptos en situaciones variadas y diferentes, lo que constituye la base de una correcta formulación diagnóstica, sea esta de una enfermedad de una situación epidemiológica e incluso de los conflictos interpersonales. Para ello ya enfatizamos lo importantes que son el uso de las TICs y la simulación entre otros.

2. Aprendizaje formativo: corresponde al desarrollo de actitudes y de una ética pertinente a su profesión. Representa el aprender a conducirse de una forma tal que comprenda los códigos de conducta esperados por su gremio para ser reconocido como tal por sus pares.

3. Aprendizaje transformacional: es la cúspide de la pirámide pues se refiere al desarrollo de actividades de liderazgo, condición fundamental para que sean agentes efectivos de cambio, que finalmente es lo puede llevar a la transformación de la sociedad para lograr los objetivos en salud.

Finalmente, es necesario puntualizar que existe otro elemento que se introducido en el quehacer de la sociedad y sin lugar a dudas en la educación y en la salud y es la globalización. Las fronteras de los países 
fueron derribadas por la comunicación virtual y las redes sociales. Hoy los ciudadanos pertenecen al mundo y existe una gran apetencia por vivir otras realidades. Esto se ha visto reflejado en la movilización de estudiantes que buscan experiencias fuera de las fronteras de su pais y las universidades han generado fuertes direcciones de relaciones internacionales y departamentos de Global Health.

Concluyendo se perciben grandes cambios en el futuro, algunos de los cuales están ya en marcha y si no tomamos en cuenta estas señales, quedaremos obsoletos en la forma de preparar profesionales para el mañana tanto en el área de la salud como en cualquier otra.

Dr. Pedro Uribe J. Rector

Universidad Andrés Bello puribe@unab.cl

\section{Referencias seleccionadas}

1. Frenk J, Chen L, Bhutta Z, Cohen J, Crisp N, Evans $\mathrm{T}$, Fineberg $\mathrm{H}$, et al. Health professionals for a new century: transforming education to strengthen health systems inan interdependent world. The Lancet 2010; 376 (9756):1923-58.

2. Frenk J. Globalization and health: the role of knowledge in an interdependent world. David E Barmes Global Health Lecture. Bethesda: National Institution of Health, 2009.

3. Institute of Medicine. The future of the public's health in the $21^{\text {st }}$ century. Washington: National Academy Press, 2002.

4. UK General Medical Council. Tomorrow's doctors: outcomes andstandards for undergraduate medical education. London: General Medical Council, 2009.
5. The Association of Faculties of Medicine of Canada. The future of medical education in Canada (FMEC): a collective vision for MDeducation. Ottawa: The Association of Faculties of Medicine of Canada, 2010.

6. Haidet P, O'Malley KJ, Richards B. An initial experience with "team learning" in medical education. Acad Med 2002;77:40-4.

\section{Links de interés}

1. https://www.youtube.com/watch? $\mathrm{v}=\mathrm{WwslBPj} 8 \mathrm{GgI}$

2. http://www.scoop.it/t/brain-development-learningconference-2013/

3. http://www.ted.com/talks/salman_khan_let_s_use_video_to_reinvent_education

4. https://www.youtube.com/watch? $\mathrm{v}=$ zpcEpmNbHds 